\title{
2(3H)-Furanones as synthons for polyamides of 1,3-diazines and 1,3,5-triazines
}

\author{
Abdel-Sattar S. Hamad Elgazwy, ${ }^{\text {a }}$ Hoda T. Zaky ${ }^{\text {, }}$ Mansoura I. Mohamed, \\ and Nadia G. Kandile ${ }^{b}$ \\ $a^{*}$ Department of Chemistry, Faculty of Science, University of Ain Shams, Abbassia 11566, \\ Cairo, Egypt \\ ${ }^{b}$ Department of Chemistry, Faculty of Girls, University of Ain Shams, Heliopolis 11757, \\ Cairo, Egypt \\ E-mail: aelgazwy@yahoo.com
}

\begin{abstract}
2(3H)-Furanones 3a-d having an exocyclic double bond and an $\mathrm{N}$-acetylisation nucleus were converted into novel polyamides of pyrimidine derivatives via reaction with nitrogen nucleophiles. NMR, GC/MS and chemical evidence confirmed all proposed structures.
\end{abstract}

Keywords: 2(3H)-Furanones, polyamide, diazines, and triazines

\section{Introduction}

The rapid assembly of "drug-like" core templates is fundamental to the discovery phase of many medicinal chemistry programs. If the syntheses of these core templates will allow for their ready functionalization, a meaningful SAR study can be efficiently conducted. In addition to ready assembly and core functionalization, the core template should have relatively low molecular weight and $\log$ P. [1,2] This will allow room for the molecule to grow as functionalization is incorporated, thus allowing the targeting of the many essential criteria of drug candidates including selectivity, potency, and efficacy. We identified compounds 5a-d, 6a-d, 8a-d, and 10ad as attractive molecular scaffolds for our medicinal chemistry program since both platforms are relatively novel in the patent literature, compatible with our pharmacophore model and have relatively low starting molecular weights and calculated $\log P$ values. Over the past two decades, 2(3H)-furanones have attracted strong interest from our group [3-8], because of possible applications of the corresponding biologically active compounds $[9,10]$. Nitrogen heterocycles constitute an important class of natural and non-natural products many of which exhibit useful biological activity [11-14]. Isatin 1, and a number of its derivatives, possess a reactive ketocarbonyl group that readily undergoes condensation reactions under mild conditions [15a]. It was 
therefore speculated that isatin would be a suitable electrophilic component for the BaylisHillman reaction [15b] and a precursor for the synthesis of other heterocycles [16-19]. Substituted isatins are found in plants such as melosatin alkaloids obtained from the caribbean tumorigenic plant melochia tomentosa [20-22] as well as from fungi, 6-(3'-methylbuten-2'yl)isatin was isolated from streptomyces albus [23] and 5-(3'-methylbuten-2'-yl)isatin from chaetomium globosum [24]. The interest in isatin derivatives stems from their pharmacological [25-26] and industrial applications [27, 28]. The present invention broadly relates to racemic compounds and their pharmaceutically acceptable salts [9], useful for treating a pathology in which reactive oxygen species and inflammatory mediators are contributing deleterious factors, such as acute or chronic inflammatory disorders, for example, asthma, rheumatoid arthritis, inflammatory bowel disease, and acute respiratory distress syndrome; neurodegenerative disorders, such as Alzheimer disease, Parkinson disease, amyotrophic lateral sclerosis, traumatic brain injury and multiple sclerosis; cardiovascular diseases, such as atherosclerosis; viral diseases, such as AIDS; skin disease, such as psoriasis; sunburn and premature aging; and eye diseases, such as glaucoma, cataract, senile macular degeneration, inflammatory eye conditions, trauma, post-traumatic eye disorders, diabetic retinopathy and eye infections.

\section{Results and Discussion}

Previously, 2(3H)-furanones [7] and 2(5H)-furanones [10] have proven to be good synthons for different, highly biological active heterocyclic compounds. Recently, 2(3H)-furanones 3a-d bearing the indole nucleus were converted into amides, pyrrolones, and imidazoles [8]. Several attempts to modify the functional groups in furanones have been made; e.g. transformation of carbonyl groups into the corresponding $\mathrm{C}=\mathrm{S}$ moieties using Lawesson's reagent [7]. As part of our previous investigation on 2-(3H)-furanones [3-7], the preparation of (3E)-1-acetyl-3(5-aryl2-oxofuran-3(2H)-ylidene)-1,3-dihydro-2H-indol-2-ones 3a-d was described by one of us [8]. This was consistent to the result described by Long et al.who studied monosubstituted 3methyleneoxindoles and detected for most of them only the E-isomer [10]. We haved examined the reactivity of 2(3H)-furanones 3a-d towards some nitrogen nucleophiles [5,6,8].

We are pleased to report herein a facile method for the synthesis of mono and diamides of biologically important pyrimidines 5a-d and 6a-d via reaction with one and two mole equivalents of 2(3H)-furanones 3a-d and with one mole equivalent of trimethoprime $\mathbf{2}$ in ethanol in good to excellent yields (64-80\%). There have not been reports of the synthesis of the ring systems 5a-d and 6a-d in the chemical literature. The reaction of 2(3H)-furanones 3a-d with trimethoprime 4 in 1:1 mole ratio, refluxing in ethanol over $12 \mathrm{~h}$, afforded monoamido 1,3diazines 3a-d in 64-80\% yield (Scheme 1). In contrast, the 1,3-diazines 3a-d were isolated in very low yield (10-15\%) when the reaction took place in a non-protic solvent. However, reaction of 2(3H)-furanones 3a-d with trimethoprime 4 in a 2:1 mole ratio provided the diamido 1,3- 
diazines 6a-d in 53-62\% yields. The latter, 6a-d, remain under our investigation and have anticipated biological activity.

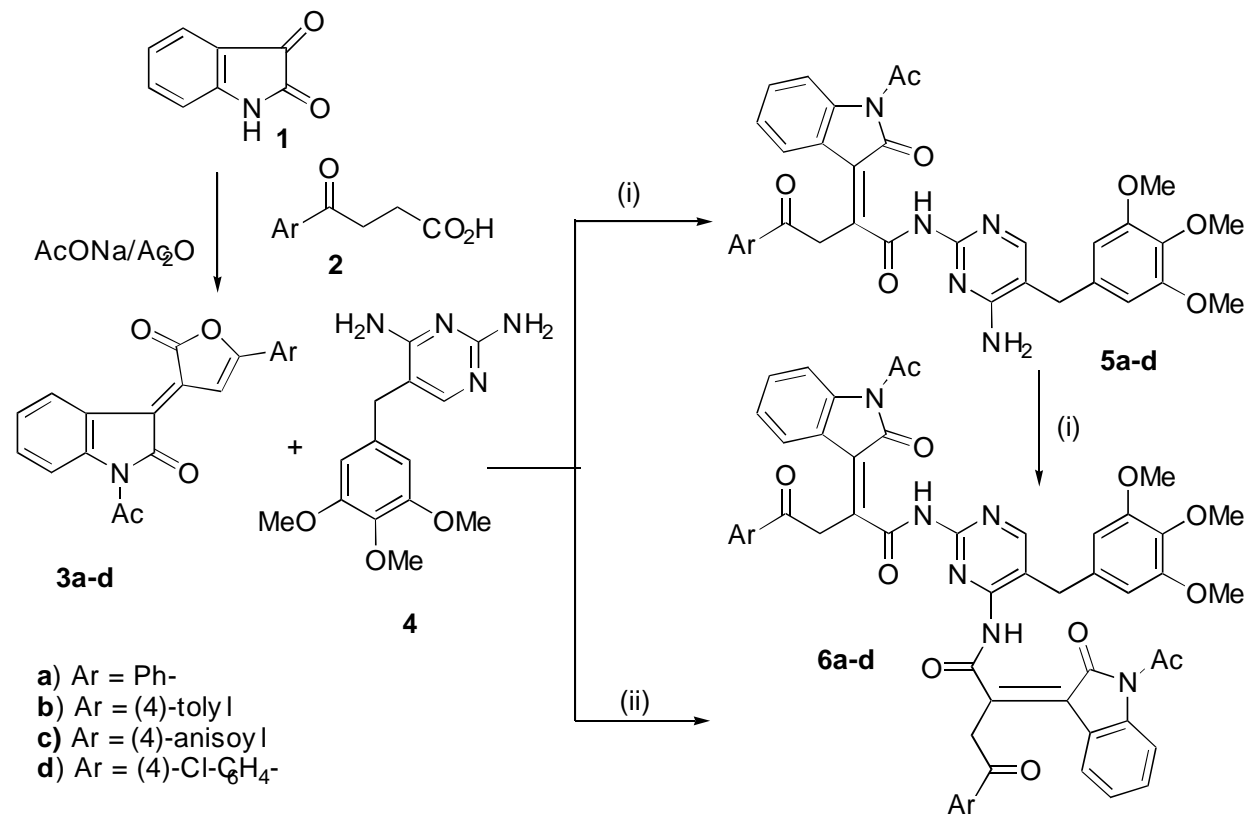

Scheme 1. Reagent and conditions: i)Addition of 1:1 molar ratio of furanones 3a-d into trimethoprime 4 in EtOH/refluxing for 12 hours; ii) Addition of 2:1 molar ratio of furanones 3a-d into trimethoprime 4 in EtOH/refluxing for 12 hours.

The 2(3H)-furanones 3a-d were treated neat with a molar equivalent of melamine 7 (1:1 mole ratio) and heating above the melting point over 2-3 h afforded monoamides 8a-d in low yield (22-35\%). These products 8a-d were isolated in similar or even better yields (36-50\%) when the reaction took place in a protic solvent $(\mathrm{EtOH})$ by ring opening (Scheme 1). However, when the reaction was repeated by adding two mole equivalents of 3a-d and one mole equivalent of melamine 7 (2:1 mole ratio) the corresponding melamides 9a-d were obtained, which were formed also by refluxing 8a-d with one mole of 3a-d for 6-12 hours. Since 9a-d are the penultimate intermediates, it follows that the utility of this reaction will depend on their ease of preparation. The route described has some limitations in that it requires multiple steps as well as pure starting materials or reaction conditions that limit the ultimate utility of the reaction. Similar reaction conditions were repeated carefully by adding three mole equivalents of 3a-d into one mole equivalent of melamine 7 (3:1 mole ratio) in refluxing ethanol to give melamides 10a-d in 35-38 \% yield. The latter were obtained also by refluxing 9a-d with one mole equivalent of 3a-d for 6-12 hours. 


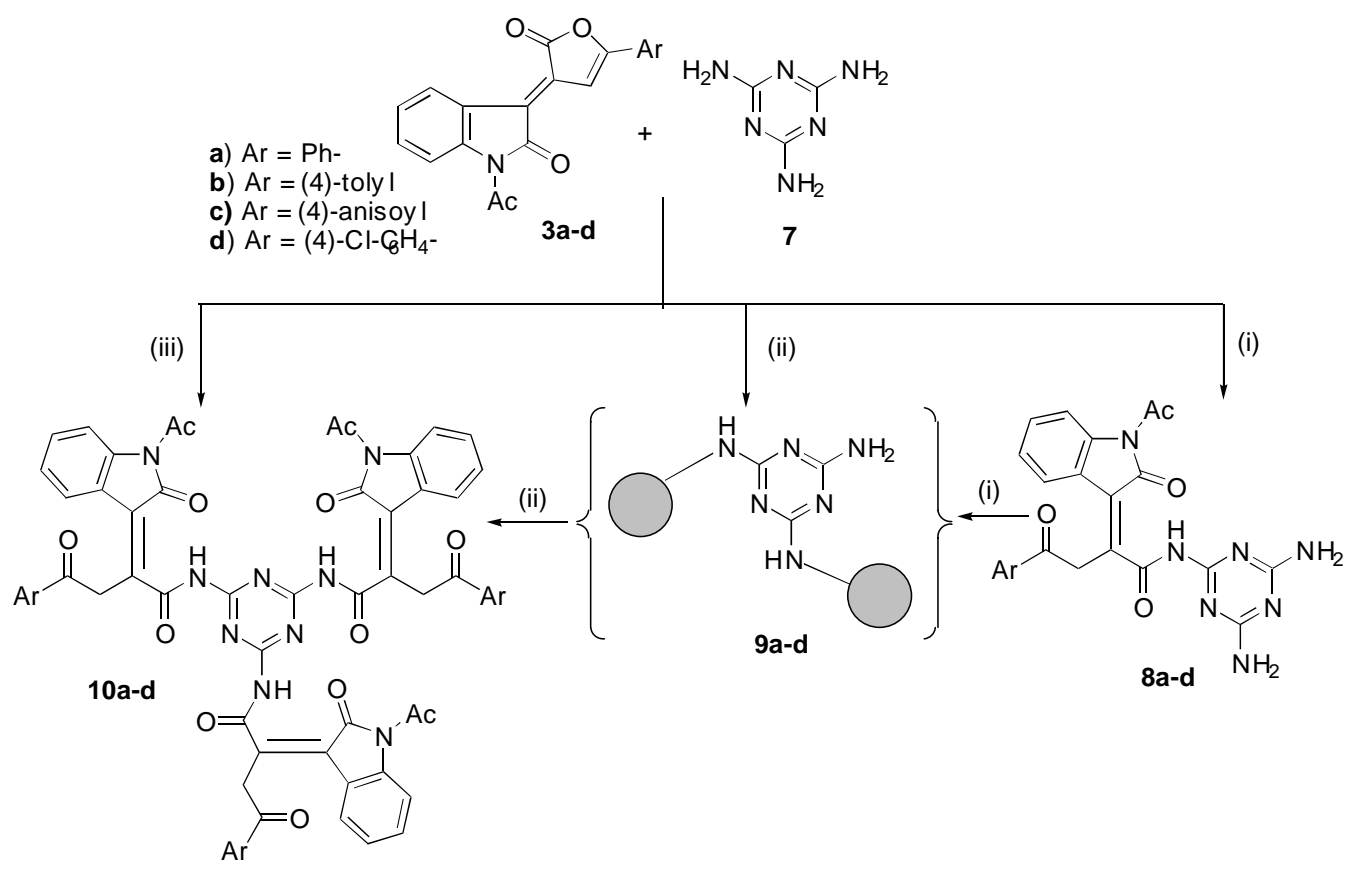

Scheme 2. Reagent and conditions: i) Addition of one molar equivalent of furanones 3a-d into melamine 7 (1:1 molar ratio) in EtOH/refluxing for 12 hours; ii) Addition of two fold molar equivalents of furanones 3a-d into melamine 7 (2:1 molar ratio) in EtOH/refluxing for 12 hours; iii) Addition of three fold molar equivalent of furanones 3a-d into melamine 7 (3:1 molar ratio) in EtOH/refluxing for 12 hours.

\section{Biological activity}

Compounds 5a-d, 6a-d, 8a-d, and 10a-d remain under our investigation and show promising biological activities against Neurodegenerative disorders (Alzheimer disease), Cardiovascular diseases (atherosclerosis) and Viral diseases (AIDS). The findings from these latter studies will be published elsewhere.

\section{Experimental Section}

General Procedures. Melting points were determined on a Boetius hot-stage apparatus and are uncorrected. Chromatographic separations were performed using silica gel (Merck, 70-230 mesh). Thin-layer chromatography was carried out on Macherey-Nagel precoated silica plates (0.25 mm layer thickness). ${ }^{1} \mathrm{H}$ NMR and ${ }^{13} \mathrm{C}$ NMR spectra were recorded with a Gemini 200 spectrometer in $\mathrm{CDCl}_{3}$ solution unless otherwise specified (internal standard TMS). Mass spectra were taken on a VG Trio-2 and Gc/Ms-QPL000EX (EI, $70 \mathrm{eV}$ ) apparatus. IR spectra were recorded with a Perkin-Elmer 1430 instrument in KBr disks. M-H-W Laboratories (Phoenix Az) 
and the Microanalytical center of Cairo and Ain Shams Universities performed elemental analyses.

General procedure for 3a-d. 2-(3H)-Furanones (3a-d) were prepared following the literature method [24,27], via condensation of isatin 1 (1.47 gm, $0.01 \mathrm{~mol})$ with 3-aroylpropionic acids 2 (0.01 mole) in acetic anhydride $(10 \mathrm{~mL})$ and fused sodium acetate $(0.03 \mathrm{~mol})$ under Perkin conditions. The corresponding $E$-isomers were obtained as the sole products, with no detectable amount of the Z-isomers being identified by TLC and ${ }^{1} \mathrm{H}$ NMR. The reddish green precipitate was collected by filtration and recrystalized from acetic acid to give 3a-d.

General procedure for $5 a-d$. To a suspension of 3a-d $(0.1 \mathrm{~mol})$ in ethanol $(30 \mathrm{~mL})$, was added trimethoprime 4 (0.1 mol) and the reaction mixture was refluxed for 6-12 hours. After cooling, the reddish brown precipitate was collected by filtration and recrystalized from toluene to give 5a-d.

2-([(2E)-2-(1-Acetyl-2-oxo-1,2-dihydro-3H-indol-3-ylidene)--4-oxo-4-phenylbutanoyl] amino\}-5-(3,4,5-trimethoxybenzyl)-4-aminopyrimidine (5a). Brown crystals $2.07 \mathrm{~g}, 64.17$ \% yield, mp >300 ${ }^{\circ} \mathrm{C}$; IR (KBr pellet): $3653.14-3751.9$ ((s), 3429.4 (broad band) for ( $\mathrm{NH}$ amide), $1772.2(\mathrm{~m}), 1727.4(\mathrm{v})$, for $(\mathrm{C}=\mathrm{O})$ of ketone 1672.6 (very sharp) for cyclic oxopyroline ring and $\mathrm{N}-\mathrm{COCH}_{3}, 1618.5$ (s), for (C=O) of amide, 1585.3 (S), $1530.4(\mathrm{~s})$, for (C=N), 1487.7 (s), 1481.0 (s), 1348.5, 760.9 (s) cm ; $^{-1}$ LRMS (EI): m/z 620 [(M+- 1), 0.27)], 510 (0.33), 417 (0.25), 289 (10.70), 261 (9.97), 156 (9.80), 105 (20.35), 101 (15.83), 77 (100), 76 (14.44), 75 (10.07), 74 (14), 50 (55.05); GC: $t_{R}=7.611 \mathrm{~min}$; column: DB-5 $6 \mathrm{~m} \mathrm{x} 0.01 \mathrm{~mm}+1 \mathrm{~m}$ guard column: temp. prog: $50^{\circ} \mathrm{C} / 2 \mathrm{~min} . / 20^{\circ} \mathrm{C} / 1 \mathrm{~min} . / 325^{\circ} \mathrm{C} / 10 \mathrm{~min} ;{ }^{1} \mathrm{H} \mathrm{NMR}\left(\mathrm{CDCl}_{3}, 200 \mathrm{MHz}\right): \delta 13.61(\mathrm{~s}, 1 \mathrm{H}$, $\mathrm{CON} \underline{\mathrm{H}}$ ) , 8.88 (s, 2H, Ar- $\underline{\mathrm{H}}_{2}$ ), 8.10 (s, 1H, pyrimidine-H(6)), 7.87-7.12 (m, 11H, -Ar-H), 6.58 (s, $2 \mathrm{H}$, pyrimidine- $\left.\underline{\mathrm{C}}_{2}-\mathrm{Ar}\right), 6.34$ (s, $\left.2 \mathrm{H},-\underline{\mathrm{C}}_{2}-\mathrm{Ar}\right), 3.78$ (s, $\left.6 \mathrm{H},-\left(\mathrm{OCH}_{3}\right)_{2}\right], 3.65\left(\mathrm{~s}, 3 \mathrm{H},-\mathrm{OCH}_{3}\right)$, 2.36 (s, 3H, $\mathrm{COCH}_{3}$ ). Calcd. for $\mathrm{C}_{34} \mathrm{H}_{31} \mathrm{~N}_{5} \mathrm{O}_{7}$ (621.639); C, 65.69; H, 5.03; N, 11.27; Found: C, 65.90; H, 5.00.; N, 11.20.

\section{2-\{([(2E)-2-(1-Acetyl-2-oxo-1,2-dihydro-3H-indol-3-ylidene)-4-(4-methylphenyl)-4-} oxobutanoyl]amino\}-5(3,4,5-trimethoxybenzyl)-4-aminopyrimidine (5b). Brown crystals 2.1 g, $66.67 \%$ yield, $\mathrm{mp}>300{ }^{\circ} \mathrm{C}$; IR (KBr pellet): 3468.8 (broad band) for ( $\mathrm{NH}$ amide), 3168.6, 3107.8, 3061.0, 3022.1, 2959.9 (s), 2923.9 (v), 2856.3 (s), 1772.2 (s), 1726.2 (very sharp), for $(\mathrm{C}=\mathrm{O})$ of ketone 1672.9 (very sharp) for cyclic oxopyroline ring and $\mathrm{N}-\mathrm{COCH}_{3}, 1618.5$ (s), for $(\mathrm{C}=\mathrm{O})$ of amide, 1585.7 (S), $1566.8(\mathrm{~s})$, for $(\mathrm{C}=\mathrm{N}), 1541.8$ (s), 1463.0 (s), 1433.2 (s), 1240.6 (s), 748.3 (m) cm ${ }^{-1}$; LRMS (EI): m/z 303 [( $\left.\left.\left.\mathrm{M}^{+}-332\right), 23.20\right)\right], 271$ (2.22), 247 (2.99), 148 (9.20), 156 (28.71), 129 (5.21), 128 (22.73), 127 (12.07), 120 (12.20), 119 (100), 103 (5), 102 (12), 101 (24.39), 90.95 (4.32), 91 (90.24), 89 (34.59), 65 (35.92), 63 (19.90), 53 (4), 50 (11.75); GC: tR = 7.339 min; column: DB-5 $6 \mathrm{~m}$ x $0.01 \mathrm{~mm}+1 \mathrm{~m}$ guard column: temp. prog: $50^{\circ} \mathrm{C} / 2 \mathrm{~min} . / 20^{\circ} \mathrm{C} / 1$

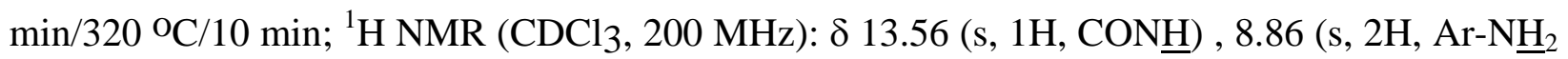
), 8.12 (s, $1 \mathrm{H}$, pyrimidine-H(6)), 8.10-7.24 (m, 10H, -Ar-H), 6.85 (s, 2H, pyrimidine- $\underline{\mathrm{H}}_{2}-\mathrm{Ar}$ ), 6.54 (s, $\left.2 \mathrm{H},-\underline{\mathrm{C}}_{2}-\mathrm{Ar}\right), 3.75$ [(s, 6H, $\left.-\left(\mathrm{OCH}_{3}\right)_{2}\right], 3.66$ (s, 3H, $\left.-\mathrm{OCH}_{3}\right), 2.67$ (s, 3H, $\left.\mathrm{COCH}_{3}\right), 2.40$ (s, 3H, $-\mathrm{CH}_{3}$ ); Calcd. for $\mathrm{C}_{35} \mathrm{H}_{33} \mathrm{~N}_{5} \mathrm{O}_{7}$ (635.23); C, 66.13; H, 5.23; N, 11.01; Found: C, 66.70; $\mathrm{H}$, 4.90; N, 11.30. 
2-\{([(2E)-2-(1-Acetyl-2-oxo-1,2-dihydro-3H-indol-3-ylidene)-4-(4-methoxyphenyl)-4oxobutanoyl]amino\}-5(3,4,5-trimethoxybenzyl)-4-aminopyrimidine (5c). Brown crystals 2.16 g, 70 \% yield, mp >300 oC, IR (KBr pellet): 3428.4 (bm) for (NH), 1773.2(s), 1723.7 (v) for $(\mathrm{C}=\mathrm{O}), 1670.1$ (v), 1606.2 (s), $1585.4(\mathrm{~m})$ and 1509 (sv) for $(\mathrm{C}=\mathrm{N}) \mathrm{cm}^{-1}$; LRMS (EI): m/z 651 ( $\left.\left.\mathrm{M}^{+}\right), 7.73\right), 650$ ( $\left.\mathrm{M}^{+}-1\right)$ (7.7), 149 (17.90), 147 (9.84), 142 (9.813), 139 (8.29), 127 (9.91), 119 (14.30), 102 (10.50), 101 (9.81), 98 (10.50), 97 (15.47), 95 (19.31), 91 (19.23), 97 (8.93), 95 (20.52), 93 (23.7), 91 (20.99), 70 (23.20), 67 (34.25), 57 (100), 55 (99.45), 51 (10.50); GC: tR = $3.219 \mathrm{~min}$; column: DB-5 $6 \mathrm{~m} \mathrm{x} 0.01 \mathrm{~mm}+1 \mathrm{~m}$ guard column: temp. prog: $50^{\circ} \mathrm{C} / 2 \mathrm{~min} . / 20^{\circ} \mathrm{C} / 1$

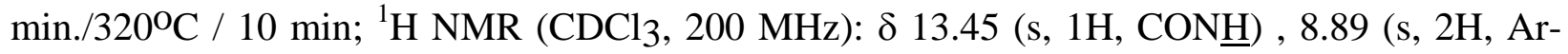
$\mathrm{NH}_{2}$ ), 8.09 (s, 1H, pyrimidine-H(6)), 8.08-7.23 (m, 10H, -Ar-H), 6.78 (s, 2H, pyrimidine- $\underline{\mathrm{H}}_{2}-$ $\mathrm{Ar}), 6.54$ (s, $\left.2 \mathrm{H},-\mathrm{CH}_{2}-\mathrm{Ar}\right), 3.79$ [(s, 6H, $\left.-\left(\mathrm{OCH}_{3}\right)_{2}\right], 3.77$ (s, 3H, $-\mathrm{OCH}_{3} 3.71$ (s, 3H, $\left.-\mathrm{OCH}_{3}\right)$, 2.58 (s, 3H, $\mathrm{COCH}_{3}$ ); Calcd. for $\mathrm{C}_{35} \mathrm{H}_{33} \mathrm{~N}_{5} \mathrm{O}_{8}$ (651.68); C, 64.50; H, 5.10; N, 10.74; Found: C, 64.30; H, 5.10; N, 10.50 .

2-\{([(2E)-2-(1-Acetyl-2-oxo-1,2-dihydro-3H-indol-3-ylidene)-4-(4-chlorophenyl)-4oxobutanoyl]amino\}-5-(3,4,5-trimethoxybenzyl)-4-aminopyrimidine (5d). Brown crystals $1.60 \mathrm{~g}, 52 \%$ yield, $\mathrm{mp}>300{ }^{\circ} \mathrm{C}$, IR (KBr pellet): 3432.6 (broad band) for (NH), 1731.2(v), 1672.3 (v) for (C=O), $1620.6(\mathrm{v}), 1589.6$ (s), 1540.1 (m) and $1486.4(\mathrm{sv}), 1094.5$ (v), 827.7 (sv),

752.4 (sv) $\mathrm{cm}^{-1}$; LRMS (EI): m/z 651 [(M+5)(6.55)], 453 (5), 384 (12), 323 (32), 232 (10), 114 (3), 113 (31.71), 112 (9.29), 111 (100), 101 (19.56), 77 (13.12), 76 (20.17), 75 (70.17), 71 (27.97), 62 (11.73), 61 (7.95), 51 (10.14); GC: tR = 7.465 min; column: DB-5 6 m x $0.01 \mathrm{~mm}+$ $1 \mathrm{~m}$ guard column: temp. prog: $50^{\circ} \mathrm{C} / 2 \mathrm{~min} . / 20^{\circ} \mathrm{C} / 1 \mathrm{~min} . / 325^{\circ} \mathrm{C} / 10 \mathrm{~min} ;{ }^{1} \mathrm{H}$ NMR (CDCl3, 200

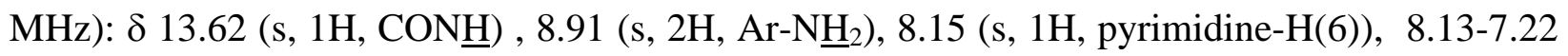
(m, 10H, -Ar-H), 6.81 (s, 2H, pyrimidine- $\left.\underline{\mathrm{C}}_{2}-\mathrm{Ar}\right), 6.54$ (s, 2H, $\left.\underline{\mathrm{H}}_{2}-\mathrm{Ar}\right), 3.80$ [(s, 6H, $\left(\mathrm{OCH}_{3}\right)_{2}$ ], 3.75 (s, 3H, $\left.-\mathrm{OCH}_{3}\right), 2.76$ (s, 3H, $\mathrm{COCH}_{3}$ ); Calcd. for $\mathrm{C}_{34} \mathrm{H}_{30} \mathrm{~N}_{5} \mathrm{O}_{7} \mathrm{Cl}(656.09)$; C, 62.24; H, 4.60; N, 10.67; Cl, 5.40; Found: C, 62.50; H, 4.60; N, 11.10.; Cl, 5.20.

General procedure for $6 \mathbf{a}-\mathbf{d}$. To a suspension of $\mathbf{3 a - d}(0.2 \mathrm{~mol})$ in ethanol $(30 \mathrm{~mL})$, was added trimethoprime $2(0.1 \mathrm{~mol})$ and the reaction mixture was refluxed for 6-12 hours. After cooling, the reddish brown precipitate was collected by filtration and recrystalized from toluene to give 6a-d.

2,4-Bis-[(2E)-2-(1-acetyl-2-oxo-1,2-dihydro-3H-indol-3-ylidene)-4-oxo-4-phenylbutanoyl] diamino-5-[(3,4,5-trimethoxybenzyl)]pyrimidine (6a). Reddish Brown crystals 0.17 g, 53 \% yield, mp 115-117 ${ }^{\circ} \mathrm{C}$; IR (KBr pellet): 3323.1, 3193.3, 2962.2, 2837.7 (bv) for (NH), 1774.0 (s), 1722.4 (s), 1662.3 (v), for (C=O) 1618.0 (s), 1618.5 (s), 1590.8 (S), 1453.1 (sv), 1425.7 (s),

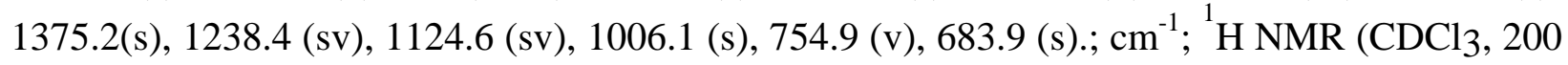

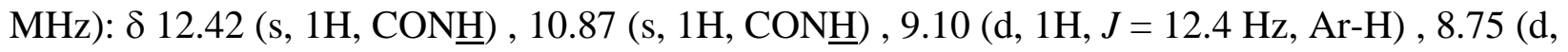
$1 \mathrm{H}, J=12.2 \mathrm{~Hz}, \mathrm{Ar}-\mathrm{H}$ ), 8.09 (s, 1H, pyrimidine-H(6)), 8.01-6.77(m, 18H, -Ar-H), 6.75 (s, 2H, $\left.\mathrm{C}_{2}\right)$ ), 6.56 (s, 2H, pyrimidine- $\left.\underline{\mathrm{H}}_{2}-\mathrm{Ar}\right), 6.44$ (s, $\left.2 \mathrm{H},-\underline{\mathrm{CH}}_{2}\right), 3.92$ (s, 3H, - $\left(\mathrm{OCH}_{3}\right), 3.72$ (s, 3H, $\mathrm{OCH}_{3}$ ), 3.56 (s, 3H, $-\mathrm{OCH}_{3}$ ), 1.94 (s, 6H, $\mathrm{COCH}_{3}$ ). Calcd. for $\mathrm{C}_{54} \mathrm{H}_{44} \mathrm{~N}_{6} \mathrm{O}_{11}$ (952.98); C, 68.06; H, 4.65; N, 8.81; Found: C, 68.50; H, 4.40.; N, 9.23. 


\section{2,4-Bis-[(2E)-2-(1-Acetyl-2-oxo-1,2-dihydro-3H-indol-3-ylidene)-4-(4-methylphenyl)-4-} oxobutanoyl]diamino-5-[(3,4,5-trimethoxybenzyl)]pyrimidine (6b). Reddish Brown crystals 0.18 g, 59 \% yield, mp 190-192 ${ }^{\circ} \mathrm{C}$; IR (KBr pellet): 3344.1 (bv), 3187.2 (bv), 2934.9 (bv), 2837.7 (bv) for (NH), 1766.2 (s), 1718.3 (s), 1662.1 (v), for (C=O) 1618.0 (s), 1618.5 (s), 1506.4 (S), 1460.2 (sv), 1423.6 (s), 1302.5 (s), 1239.0 (sv), 1125.2 (sv), 1010.6 (s), 755.2(v) cm ${ }^{-1} ;{ }^{1} \mathrm{H}$

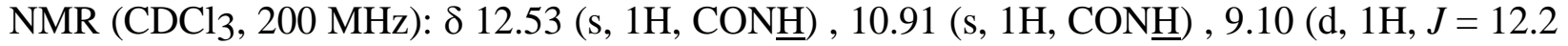
$\mathrm{Hz}, \mathrm{Ar}-\mathrm{H}$ ) , 8.70 (d, 1H, $J=12.2 \mathrm{~Hz}, \mathrm{Ar}-\mathrm{H}), 8.03$ (s, 1H, pyrimidine-H(6)), 7.88-6.77(m, 16H, Ar-H), 6.57 (s, 2H, pyrimidine- $\underline{\mathrm{C}}_{2}-\mathrm{Ar}$ ), 6.34 (s, 2H, $-\mathrm{C}_{2}-\mathrm{Ar}$ ), 5.97 (s, $2 \mathrm{H},-\underline{\mathrm{C}}_{2}-\mathrm{Ar}$ ), 3.82-3.73 [(singlet with shoulder signal, 6H, -( $\left.\left(\mathrm{OCH}_{3}\right)_{2}\right], 3.63-3.54$ (singlet with shoulder signal, 3H, $\left(\mathrm{OCH}_{3}\right), 2.36\left[\left(\mathrm{~s}, 6 \mathrm{H}, \mathrm{COCH}_{3}\right)_{2}\right], 1.92$ [(s, 6H, $\left.\left(\mathrm{CH}_{3}\right)_{2}\right]$. Calcd. for $\mathrm{C}_{56} \mathrm{H}_{48} \mathrm{~N}_{6} \mathrm{O}_{11}$ (981.03); $\mathrm{C}$, 68.56; H, 4.93; N, 8.56; Found: C, 69.00; H, 5.10; N, 8.23.

2,4-Bis-[(2E)-2-(1-Acetyl-2-oxo-1,2-dihydro-3H-indol-3-ylidene)-4-(4-methoxyphenyl)-4oxobutanoyl]diamino-5-[(3,4,5-trimethoxybenzyl)]pyrimidine (6c). Reddish Brown crystals 0.19 g, 62 \% yield, mp 110-112 ${ }^{\circ} \mathrm{C}$; IR (KBr pellet): 3344.1 (bv), 3187.2 (bv), 2934.9 (bv), 2837.7 (bv) for (NH), 1766.2 (s), 1718.3 (s), 1662.1 (v), for (C=O) 1618.0 (s), 1618.5 (s), 1506.4 (S), 1460.2 (sv), 1423.6 (s), 1302.5 (s), 1239.0 (sv), 1125.2 (sv), 1010.6 (s), 755.2(v) $\mathrm{cm}^{-1} ;{ }^{1} \mathrm{H}$

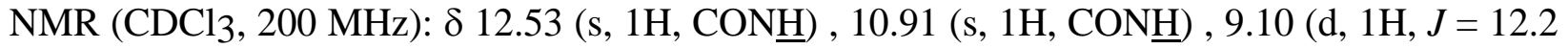
Hz, Ar-H) , 8.70 (d, 1H, $J=12.2 \mathrm{~Hz}, \mathrm{Ar}-\mathrm{H}$ ), 8.03 (s, 1H, pyrimidine-H(6)), 7.88-6.77(m, 16H, Ar-H), 6.57 (s, 2H, pyrimidine- $\left.\underline{\mathrm{H}}_{2}-\mathrm{Ar}\right), 6.34\left(\mathrm{~s}, 2 \mathrm{H},-\mathrm{C}_{2}\right), 5.97$ (s, $\left.2 \mathrm{H},-\underline{\mathrm{C}}_{2}\right), 3.82-3.73$ [(singlet with shoulder signal, 6H, -( $\left.\left(\mathrm{OCH}_{3}\right)_{2}\right], 3.63-3.54$ (singlet with shoulder signal, 3H, $\left(\mathrm{OCH}_{3}\right), 2.36$ [(s, 6H, $\left.\mathrm{COCH}_{3}\right)_{2}$ ], 1.92 [(s, 6H, $\left(\mathrm{CH}_{3}\right)_{2}$ ].); Calcd. for $\mathrm{C}_{56} \mathrm{H}_{48} \mathrm{~N}_{6} \mathrm{O}_{13}$ (1013.03); C, 66.39; H, 4.77; N, 8.29; Found: C, 66.93; H, 5.00; N, 8.79.

\section{2,4-Bis-[(2E)-2-(1-Acetyl-2-oxo-1,2-dihydro-3H-indol-3-ylidene)-4-(4-chlorophenyl)-4-} oxobutanoyl]diamino-5-[(3,4,5-trimethoxybenzyl)]pyrimidine (6d). Brown crystals $1.60 \mathrm{~g}$, 52\% yield, $\mathrm{mp}>300^{\circ} \mathrm{C}$, IR ( $\mathrm{KBr}$ pellet): 3432.6 (broad band) for (NH), 1731.2(v), 1672.3 (v) for (C=O), 1620.6 (v), $1589.6(\mathrm{~s}), 1540.1$ (m) and $1486.4(\mathrm{sv}), 1094.5$ (v), 827.7 (sv), 752.4

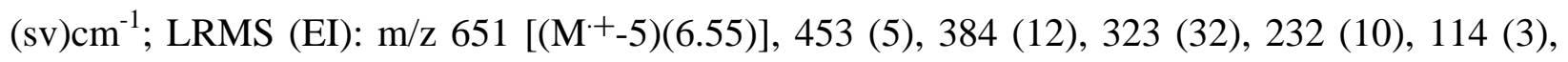
113 (31.71), 112 (9.29), 111 (100), 101 (19.56), 77 (13.12), 76 (20.17), 75 (70.17), 71 (27.97), 62 (11.73), 61 (7.95), 51 (10.14); GC: tR $=7.465 \mathrm{~min}$; column: DB-5 $6 \mathrm{~m} \mathrm{x} 0.01 \mathrm{~mm}+1 \mathrm{~m}$ guard column: temp. prog: $50^{\circ} \mathrm{C} / 2 \mathrm{~min} . / 20^{\circ} \mathrm{C} / 1 \mathrm{~min} . / 325^{\circ} \mathrm{C} / 10 \mathrm{~min} ;{ }^{1} \mathrm{H}$ NMR $\left(\mathrm{CDCl}_{3}, 200 \mathrm{MHz}\right): \delta$ 12.38 (s, $1 \mathrm{H}, \mathrm{CON} \underline{\mathrm{H}}), 10.87$ (s, $1 \mathrm{H}, \mathrm{CON} \underline{\mathrm{H}}), 9.12$ (d, $1 \mathrm{H}, J=12.2 \mathrm{~Hz}, \mathrm{Ar}-\mathrm{H}), 8.75$ (d, $1 \mathrm{H}, J=$ $12.2 \mathrm{~Hz}, \mathrm{Ar}-\mathrm{H}$ ), 8.33 (s, 1H, pyrimidine-H(7)), 8.12-6.77 (m, 18H, -Ar-H), 6.75 (s, 2H, $\mathrm{C}_{2} \mathrm{COAr}$ ), 6.56 (s, $2 \mathrm{H}$, pyrimidine- $\underline{\mathrm{H}}_{2}-\mathrm{Ar}$ ), 6.44 (s, $2 \mathrm{H},-\mathrm{C}_{2} \mathrm{COAr}$ ), 3.92 (s, $3 \mathrm{H},-\left(\mathrm{OCH}_{3}\right)$, 3.72 (s, $3 \mathrm{H},-\mathrm{OCH}_{3}$ ), 3.56 (s, $3 \mathrm{H},-\mathrm{OCH}_{3}$ ), 1.94 (s, $6 \mathrm{H}, \mathrm{COCH}_{3}$ ).; Calcd. for $\mathrm{C}_{54} \mathrm{H}_{42} \mathrm{~N}_{6} \mathrm{O}_{11} \mathrm{Cl}_{2}$ (1021.87); C, 63.47; H, 4.14; N, 8.2; Cl, 6.93; Found: C, 63.88; H, 3.90; N, 8.56.; Cl, 6.71.

\section{General procedure for $8 \mathrm{a}-\mathrm{d}$}

To a suspension of 3a-d $(0.1 \mathrm{~mol})$ in ethanol $(30 \mathrm{~mL})$, was added melamine $5(0.1 \mathrm{~mol})$ and the reaction mixture was refluxed for 6-12 hours. Afetr cooling, the semi-solid precipitate was collected by filteration and titurated with pet. ether. The solid product obtained was recrystalized from ethanol to give 8a-d. 


\section{2-[(2E)-2-(1-Acetyl-2-oxo-1,2-dihydro-3H-indol-3-ylidene)-4-oxo-4-phenylbutanoyl}

Jamino\}4,6-diamino-1,3,5-triazine (8a). Deep brown crystal $455 \mathrm{mg}, 50$ \% yield, mp 245-247 ${ }^{\circ} \mathrm{C}$; IR (KBr pellet): 3468.4--2975.2 (bm), 3429.4 (broad band) for ( $\mathrm{NH}$ amide), 1772.2 (m), $1727.4(\mathrm{v})$, for $(\mathrm{C}=\mathrm{O})$ of ketone 1655.5 (very sharp) for cyclic oxopyrroline ring and $\mathrm{N}-\mathrm{COCH}_{3}$, $1550.6(\mathrm{~s})$, for $(\mathrm{C}=\mathrm{O})$ of amide, $1463.2(\mathrm{~S}), 1375.2(\mathrm{~s})$, for $(\mathrm{C}=\mathrm{N}), 1320.6(\mathrm{~s}), 1047.8(\mathrm{~s}) \mathrm{cm}^{-1}$; LRMS (EI): m/z 456 [(M+ 1), 11.74)], 184 (83), 105 (100), 77 (94.74), 75 (10.07), 74 (14), 50 (55.05); GC: $\mathrm{tR}=7.914 \mathrm{~min}$; column: DB-5 $6 \mathrm{~m} \times 0.01 \mathrm{~mm}+1 \mathrm{~m}$ guard column: temp. prog. $50^{\circ} \mathrm{C} / 2 \mathrm{~min} . / 20^{\circ} \mathrm{C} / 1 \mathrm{~min} . / 325^{\circ} \mathrm{C} / 10 \mathrm{~min} ;{ }^{1} \mathrm{H} \mathrm{NMR}\left(\mathrm{CDCl}_{3}, 200 \mathrm{MHz}\right), \delta 8.94(\mathrm{~s}, 1 \mathrm{H},-$

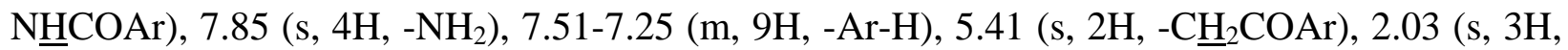
$\mathrm{COCH}_{3}$ ); Calcd. for $\mathrm{C}_{23} \mathrm{H}_{19} \mathrm{~N}_{7} \mathrm{O}_{4}$ (457.45); C, 60.39; H, 4.18; N, 21.43; Found: C, 60.50; H, 3.90.; N, 21.70.

2-[(2E)-2-(1-Acetyl-2-oxo-1,2-dihydro-3H-indol-3-ylidene)-4-(4-methylphenyl)-4oxobutanoyl]amino-4,6-diamino-1,3,5-triazine (8b). Reddish Brown crystals $454 \mathrm{mg}, 48 \%$ yield, mp 242-244 ${ }^{\circ} \mathrm{C}$; IR (KBr pellet): 3340.7 (m), 3176.2 (broad band) for ( $\mathrm{NH}$ amide), 2955.9, 2924.9 (s), 2854.8 (w), 1694.7 (vp), for ( $\mathrm{C}=\mathrm{O})$ of ketone 1616.7 (s) for cyclic oxopyrroline ring and $\mathrm{N}-\mathrm{COCH}_{3}, 1546.8(\mathrm{~s})$, for $(\mathrm{C}=\mathrm{O})$ of amide, $1501.7(\mathrm{~s}), 1462.8(\mathrm{~m})$, for $(\mathrm{C}=\mathrm{N}), 1376.2(\mathrm{~m})$, 1273.5 (s) $\mathrm{cm}^{-1}$; LRMS (EI): m/z 448 [( $\left.\left.\mathrm{M}^{+}-23\right), .50\right)$ ], 303 (17), 259 (8), 257 (2.01), 247 (5), 156 (12), 119 (100), 117 (2.9), 101 (11.55), 100 (2.17), 91 (50.02), 90 (7.03), 89 (12.01), 70 (10.75), 65 (20), 51 (10); GC: tR $=6.793 \mathrm{~min}$; column: DB-5 $6 \mathrm{~m} \mathrm{x} 0.01 \mathrm{~mm}+1 \mathrm{~m}$ guard column: temp. prog: $50^{\circ} \mathrm{C} / 2 \mathrm{~min} . / 20^{\circ} \mathrm{C} / 1 \mathrm{~min} / 300^{\circ} \mathrm{C} / 10 \mathrm{~min} ;{ }^{1} \mathrm{H} \mathrm{MR}\left(\mathrm{CDCl}_{3}, 200 \mathrm{MHz}\right): \delta 8.90$ (s, 1H, NHCO) , 8.05 (s, 4H, NH2), 7.75-6.84 (m, 8H, -Ar-H), 5.23 (s, 2H, - $\mathrm{C}_{2} \mathrm{COAr}$ ), 2.43 (s, $\left.3 \mathrm{H}, \mathrm{COCH}_{3}\right), 1.79$ (s, $3 \mathrm{H},-\mathrm{CH}_{3}$ ); Calcd. for $\mathrm{C}_{24} \mathrm{H}_{21} \mathrm{~N}_{7} \mathrm{O}_{4}$ (471.47); C, 61.14; $\mathrm{H}, 4.48 ; \mathrm{N}, 20.79$; Found: C, 61.50; H, 4.40; N, 21.00.

2-[(2E)-2-(1-Acetyl-2-oxo-1,2-dihydro-3H-indol-3-ylidene)-4-(4-methoxylphenyl)-4oxobutanoyl]amino-4,6-diamino-1,3,5-triazine (8c). Reddish Brown crystals $350 \mathrm{mg}, 36 \%$ yield, mp 262-264 ${ }^{\circ} \mathrm{C}$; IR (KBr pellet): 3344.5 (m), 3214.9 (broad band) for ( $\mathrm{NH}$ amide), 2920.4, 2847.5 (w), 1713.3 (s), 1605.3 (vp), for (C=O), 1508.7 (s), 1501.7 (s) for (C=N), 1463.5 (m), 1376.2 (m), 1253.2 (vp), 1170.8 (m) cm ${ }^{-1}$; LRMS (EI): m/z 481 [(M+ 6), .10.45)], 305.20 (12.00), 303.00 (10.90), 270.55 (11.91), 272.30 (17.91), 240.30 (15.97), 213.05 (9.70), 195.05 (11.10), 157 (10.45), 136 (19.10), 135 (100), 134 (11.10), 132 (10.45), 120 (13.13), 107 (23.13), 103 (14.93), 101 (15.97), 92 (92.84), 87 (12.00), 77 (55.21), 70 (25.37), 84 (35.07), 63 (40.51), 51 (15.87); GC: tR $=9.133 \mathrm{~min}$; column: DB-5 $6 \mathrm{~m} \mathrm{x} 0.01 \mathrm{~mm}+1 \mathrm{~m}$ guard column: temp. prog: $50^{\circ} \mathrm{C} / 2 \mathrm{~min} . / 20^{\circ} \mathrm{C} / 1 \mathrm{~min} / 320^{\circ} \mathrm{C} / 10 \mathrm{~min} ;{ }^{1} \mathrm{H}$ MR (CDCl3, $\left.200 \mathrm{MHz}\right): \delta 8.89$ (s, $\left.1 \mathrm{H}, \mathrm{NHCO}\right)$, , 8.08 (s, 4H, NH $\mathrm{N}_{2}$ ), 7.97-6.84 (m, 8H, -Ar-H), 5.23 (s, 2H, - $\underline{\mathrm{C}}_{2} \mathrm{COAr}$ ), 3.89 (s, 3H, $\mathrm{OCH}_{3}$ ), 2.42 (s, 3H, $\mathrm{COCH}_{3}$ ); Calcd. for $\mathrm{C}_{24} \mathrm{H}_{21} \mathrm{~N}_{7} \mathrm{O}_{5}$ (487.47); C, 59.13; H, 4.34; N, 20.11; Found: C, 59.40; H, 4.30; N, 19.90 .

2-[(2E)-2-(1-Acetyl-2-oxo-1,2-dihydro-3H-indol-3-ylidene)-4-(4-chlorophenyl)-4-

oxobutanoyl]amino-4,6-diamino-1,3,5-triazine (8d). Deep Brown crystals $440 \mathrm{mg}, 45 \%$ yield, mp 244-246 ${ }^{\circ} \mathrm{C}$; IR (KBr pellet): 3469.6 .5 (w), 3420.7 (m), 3337.8 (s), 3137.9 (broad band) for 
(NH amide), 2922.1, 2847.5 (w), 1648.7 (vp), for (C=O), 1549.4 (s), 1463.9 (s) for (C=N), 1439.0 (m), 1370.8 (m), 1317.3 (vp), 1221.0 (m) cm ${ }^{-1}$; LRMS (EI): m/z 490.50 [( $\left.\left.{ }^{+}-1\right), 17.24\right)$ ], 168 (42.52), 155 (21.04), 126 (100), 125 (29.44), 111 (40.23), 83 (83.22), 75 (22.90), 74 (24.14), 68 (45.90), 63 (22.90), 62 (25.29), 55 (21.94), 53 (27.50), 52 (26.44), 51 (31.03); GC: tR = 6.727 min; column: DB-5 $6 \mathrm{~m}$ x $0.01 \mathrm{~mm}+1 \mathrm{~m}$ guard column: temp. prog: 500 $\mathrm{C} / 2 \mathrm{~min} . / 20^{\circ} \mathrm{C} / 1$

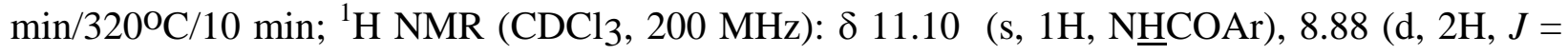
$\left.7.8 \mathrm{~Hz}, \mathrm{Cl}_{-} \mathrm{C}_{6} \underline{\mathrm{H}}_{4}-\right)$ ), 8.07 (s, $\left.4 \mathrm{H}, \underline{\mathrm{N}}_{2}\right), 7.77$ (t, 1H, $J=8.2 \mathrm{~Hz}$, pyrimidine-H), 7.43 (m, 3H), 6.84 (d, $2 \mathrm{H}, J=7.8 \mathrm{~Hz}, \mathrm{Cl}-\mathrm{C}_{6} \underline{\mathrm{H}}_{4}-$ ), 5.47 (s, $2 \mathrm{H},-\mathrm{ArCOC}_{2}$ ), 2.09 (s, 3H, $\mathrm{COCH}_{3}$ ); Calcd. for $\mathrm{C}_{23} \mathrm{H}_{18} \mathrm{~N}_{7} \mathrm{O}_{4} \mathrm{Cl}(491.89) ; \mathrm{C}, 56.16 ; \mathrm{H}, 3.68 ; \mathrm{N}, 19.93$; Cl, 7.20.; Found: C, 56.50; H, 3.50; N, 20.50, Cl, 7.60.

\section{General procedure for $10 \mathrm{a}-\mathrm{d}$}

To a suspension of 3a-d $(0.3 \mathrm{~mol})$ in ethanol $(30 \mathrm{~mL})$, was added melamine $7(0.1 \mathrm{~mol})$ and the reaction mixture was refluxed for 6-12 hours. After cooling. the semi-solid precipitate was collected by filtration and triturated with pet. ether. The solid product obtained was recrystalized from ethanol to give 10a-d.

2,4,6-Tri\{-[(2E)-2-(1-acetyl-2-oxo-1,2-dihydro-3H-indol-3-ylidene)-4-oxo-4-phenylbutanoyl] amino-1,3,5-triazine (10a). Brown crystal 1.20 gm, $35 \%$ yield, mp 105-107 ${ }^{\circ} \mathrm{C}$; IR (KBr pellet): 33343.4-3157.2 (broad band) for ( $\mathrm{NH}$ amide), 1704.0 (bs), 1619.8 (m), for (C=O) of ketone 1542.8 (w) for cyclic oxopyrroline ring and $\mathrm{N}-\mathrm{COCH}_{3}, 1448.1(\mathrm{~s})$, for $(\mathrm{C}=\mathrm{O})$ of amide, 1371.8 (S), 1340.6 (s), for (C=N), 1278.1 (s), 1241.4 .8 (s), 1174.6, 1101.4, 1017.5, 914.8, 887.9, 756.6 (v), $685.9 \mathrm{~cm}^{-1}$; ${ }^{1} \mathrm{H}$ NMR (CDCl3, $200 \mathrm{MHz} \delta 12.53$ (s, 1H, ArCONH), 10.92 (s, 2H, $\operatorname{ArCON} \underline{H}$ ), 8.71 (d, $\left.1 \mathrm{H}, J=7.7 \mathrm{~Hz}, \mathrm{Ar}_{-} \underline{\mathrm{H}}_{7}\right), 8.20-7.85$ (m, 26H, Ar-H), 6.55 (s, 2H, $\mathrm{PhCH}_{2}$ ), 6.43 [s, 4H, 2( $\left.\mathrm{PhCH}_{2-}\right)$ ], 2.08 (s, 3H, $\left.\mathrm{COCH}_{3}\right), 2.04$ (s, 3H, $\left.\mathrm{COCH}_{3}\right), 1.91-1.90$ (s, 3H, $\left.\mathrm{COCH}_{3}\right)$; Calcd. for $\mathrm{C}_{63} \mathrm{H}_{45} \mathrm{~N}_{9} \mathrm{O}_{12}$ (1119.10); C, 67.61; H, 3.96; N, 11.26; Found: C, 68.03; H, 3.82.; N, 11.72 .

2,4,6-Tri\{[(2E)-2-(1-acetyl-2-oxo-1,2-dihydro-3H-indol-3-ylidene)-4-(4-methylphenyl)-4oxobutanoyl]\}amino-1,3,5-triazine (10b). Dark brown crystal 1.30 gm, 37\% yield, mp; (decomp. $142{ }^{\circ} \mathrm{C}$ ) 170-172 ${ }^{\circ} \mathrm{C}$; IR (KBr pellet): 3469.3 (w), 3418.7 (m), 3335.4 (bb), 3132.7, (broad band) for (NH amide), 1717.1 (bs), $1690.2(\mathrm{~m})$, for $(\mathrm{C}=\mathrm{O})$ of ketone 1658.0.8 (w) for cyclic oxopyrroline ring and $\mathrm{N}-\mathrm{COCH}_{3}, 1619.9$ (s), for $(\mathrm{C}=\mathrm{O})$ of amide, 1580.7, 1546.9, 1466.5, 1435.5, 1373.7 (S), 1339.3 (s), for (C=N), 1301.2 (s), 1239.2 (s), 1181.1, 1017.9, 815.7, 754.1 $\mathrm{cm}^{-1}$; ${ }^{1} \mathrm{H}$ NMR $\left(\mathrm{CDCl}_{3}, 200 \mathrm{MHz} \delta 12.51\right.$ (s, 1H, ArCONH), 10.91 (s, 2H, ArCONH), 9.08 (d, $4 \mathrm{H}, J=7.7 \mathrm{~Hz}, \mathrm{CH}_{3}-\mathrm{C}_{6} \underline{\mathrm{H}}_{4}-$ ), 8.72 (d, $1 \mathrm{H}, J=9.6 \mathrm{~Hz}, \mathrm{Ar}-\underline{\mathrm{H}}_{7}$ ), 8.04-6.90 (m, 19H, Ar-H), 6.54 (s,

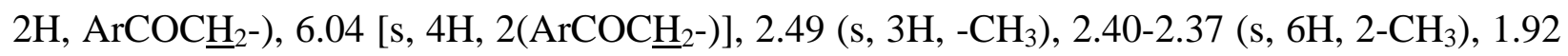
[(s, 9H, 3(-COCH$)$ ]; Calcd. for $\mathrm{C}_{66} \mathrm{H}_{51} \mathrm{~N}_{9} \mathrm{O}_{12}$ (1162.19); C, 68.21; H, 4.42; N, 10.84; Found: C, 68.67; H, 4.09.; N, 11.20.

2,4,6-Tri-\{[(2E)-2-(1-acetyl-2-oxo-1,2-dihydro-3H-indol-3-ylidene)-4-(4-methoxylphenyl)-4oxobutanoyl]\}amino-1,3,5-triazine (10c). Black crystal 1.40 gm, 38.6\% yield, mp. 103-105 ${ }^{\circ} \mathrm{C}$; IR (KBr pellet): 3339.8 (bb), 3131.2 (broad band) for (NH amide), 1768.1 (bs), 1691.3 (m), for 
$(\mathrm{C}=\mathrm{O})$ of ketone $1603.3(\mathrm{w})$ for cyclic oxopyrroline ring and $\mathrm{N}-\mathrm{COCH}_{3}, 1506.1(\mathrm{~s})$, for $(\mathrm{C}=\mathrm{O})$ of amide, 1463.4, for (C=N), 1261.8 (s), 1239.9, 1170.4 (s), 1102.3, 1020.6, 828.8, $759.6 \mathrm{~cm}^{-1}$;

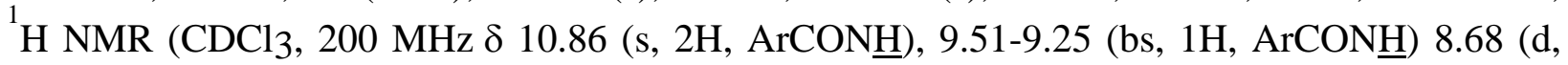
$\left.4 \mathrm{H}, J=7.62 \mathrm{~Hz}, \mathrm{CH}_{3} \mathrm{O}-\mathrm{C}_{6} \underline{\mathrm{H}}_{4}-\right), 7.96-7.83$ (m, 8H, Ar-H), 7.37-6.85 (m, 15H, Ar-H), 6.55 ( s, $2 \mathrm{H},\left(-\mathrm{CH}_{2}-\right)$ ], $6.40\left(\mathrm{~s}, 4 \mathrm{H}, 2\left(-\mathrm{CH}_{2}-\right)\right.$ ], 3.93 (s, $\left.3 \mathrm{H},-\mathrm{O} \mathrm{CH}_{3}\right), 3.86$ (s, 3H, $\left.-\mathrm{O} \mathrm{CH}_{3}\right), 3.78$ (s, 3H, -O $\mathrm{CH}_{3}$ ), 2.04 (s, $3 \mathrm{H}, \mathrm{COCH}_{3}$ ), 2.02 (s, 3H, $\mathrm{COCH}_{3}$ ), 1.92 (s, 3H, $\mathrm{COCH}_{3}$ ); Calcd. for $\mathrm{C}_{66} \mathrm{H}_{51} \mathrm{~N}_{9} \mathrm{O}_{12}$ (1210.14); C, 65.51; H, 4.22; N, 10.42; Found: C, 66.00; H, 3.99; N, 10.00.

\section{2,4,6-Tri-[(2E)-2-(1-acetyl-2-oxo-1,2-dihydro-3H-indol-3-ylidene)-4-(4-chlorophenyl)-4-}

oxobutanoyl]amino-1,3,5-triazine (10d). Brown crystal 1.30 gm, 35\% yield, mp. 137-139 ${ }^{\circ} \mathrm{C}$; IR (KBr pellet): 3342.7 (bb), 3134.26 (broad band) for (NH amide), 1679.0 (bs), 1623.3 (m), for $(\mathrm{C}=\mathrm{O})$ of ketone $1585.8(\mathrm{w})$ for cyclic oxopyrroline ring and $\mathrm{N}-\mathrm{COCH}_{3}, 1544.0(\mathrm{~s})$, for $(\mathrm{C}=\mathrm{O})$ of amide, 1466.2, for (C=N), $1340.3(\mathrm{~s}), 1239.9,1190.9(\mathrm{~s}), 1092.7,1011.8,826.5,754.7(\mathrm{~s}) \mathrm{cm}^{-}$

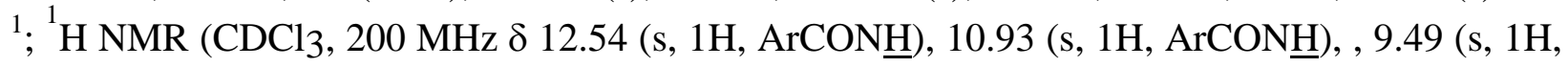
$\operatorname{ArCON} \underline{H}), 9.08$ (d, $\left.1 \mathrm{H}, J=9.2 \mathrm{~Hz}, \operatorname{Ar}-\underline{H}_{7}\right), 8.71-6.92$ (m, 23H, Ar-H), 6.59 ( s, $2 \mathrm{H},-\mathrm{CH}_{2}-$ ), 6.39 ( s, 4H, 2(- $\left.-\mathrm{CH}_{2}-\right)$ ], 2.01 (s, 3H, $\left.\mathrm{COCH}_{3}\right), 1.91$ (s, 3H, $\left.\mathrm{COCH}_{3}\right), 1.90$ (s, 3H, $\mathrm{COCH}_{3}$ ); Calcd. for $\mathrm{C}_{63} \mathrm{H}_{42} \mathrm{~N}_{9} \mathrm{O}_{12} \mathrm{Cl}_{3}$ (1223.44); C, 61.85; H, 3.46; N, 10.30; Cl, 8.69; Found: C, 62.10; H, 3.23; N, 10.63; Cl, 9.00 .

\section{Acknowledgements}

Support of this work by the Department of Chemistry, Faculty of Girls, Ain Shams University is highly appreciated.

\section{References and Footnotes}

1. Lipinski, C. A.; Lombardo, F.; Dominy, B. W.; Feeney, P. J. Adv. Drug Delivery Rev. 1997, 23, 3.

2. Teague, S. J.; Davis, A. M.; Leeson, P. D.; Oprea, T. Angew. Chem., Int. Ed. 1999, 38, 3743.

3. Hamad, A-S. S.; Hashem, A. I. J. Heterocycl. Chem. 2002, 39, 1.

4. Hamad, A-S. S.; Hashem, A. I. Molecules 2000, 5, 895.

5. Hamad, A-S. S.; Hashem, A. I. Acta. Chim. Solv. 2001, 48, 431.

6. (a) Hamad, A-S. S.; Derbala H. A.; Elsayed W. A.; Hashem, A. I. Acta. Chim. Solv. 2001, 48, 417. (b) Hashem, A. I. J. Prakt. Chem. 1977, 319, 689.

7. (a) Hamad, A-S. S.; Hashem, A. I.; El-Kafrawy, A. F.; Saad, M. M. Phosphorus, Sulfur and Silicon. 2000, 159, 157. (b) Hashem, A. I.; Senning, A. Adv. Heterocycl. Chem. 1999, 73, 275. (c) Gammill, R. B.; Wilson, C. A.; Bryson, T. A. Synth. Commun. 1975, 5, 245.

8. Hamad Elgazwy, A-S. S.; Zaky, H. T.; Mohamed, M. I.; Kandile, N. G. Heteroatom Chem. 2003, 14, 434. 
9. Appere, G.; Erdelmeier, I; Banissi, C.; Moutet, M.; Hopper, A. U.S. Patent, 6,265,436, 2001.

10. a) Long, D. R.; Richards, C. G.; Ross, M. S. F. J. Heterocycl. Chem. 1978, 15, 633.; b) Chiaccho, U.; Piperno, A.; Rescifina, A.; Romeo, G.; Uccella, N. Tetrahedron 1998, 54, 5695.; c) Hoye, T. R.; Hanson, P. R. Tetrahedron Lett. 1993, 34, 5043.

11. Flitsch, W. Comprehensive Heterocyclic Chemistry, Katritzky, A. R.; Rees, C. W. Pergamon: Oxford, 1984; Vol. 3, pp 443.

12. Swinbourne, J. F.; Hunt, H. J.; Klinkert, G. Adv. Heterocycl. Chem. 1987, 23, 103.

13. Flitsch, W. Comprehensive Heterocyclic Chemistry, Katritzky, A. R.; Rees, C. W.; Scriven, E. F. V., Eds.; Pergamon: Oxford, 1996; Chapter. 8.01, pp 1-24.

14. Gonzalez, F. B. Comprehensive Heterocyclic Chemistry, Katritzky, A. R.; Rees, C. W.; Scriven, E. F. V., Eds.; Pergamon: Oxford, 1996; Chapter 8.36, pp 967-1022.

15. (a) Da Silva, F. S. M.; Garden, S. J.; Pinto, A. C. J. Braz. Chem. Soc 2001, 12, 273. (b) Garden, S. J.; Skakl, J. M. S. Tetrahedron Lett. 2002, 43, 1969.

16. Sumpter, W. C. Chem. Rev. 1954, 34, 407.

17. Propp, F. D. Adv. Heterocyclic. Chem. 1975, 18, 1.

18. Shvekhgeimer, M. G. A. Chem. Heterocyl. Cpd (Engl. Trans,) 1996, 32, 249.

19. Da Silva, F. S. M.; Garden, S. J.; Pinto, A. C. http://preprint.chemweb.com/orgchem/ (2000).

20. Kapodia, G. J.; Shukla, Y. N.; Choudhury, B. K.; Basak, S. P.; Fales, H. M.; Sokoloski, E. A. J. Chem. Soc., Chem. Commun. 1977, 535.

21. Kapodia, G. J.; Shukla, Y. N.; Basak, S. P.; Sokoloski, E. A.; Fales, H. M. Tetrahedron 1980, 36, 2441.

22. Kapodia, G. J.; Shukla, Y. N. Planta Med. 1993, 59, 568.

23. (a) Grafe, U.; Radics, L. J. Antibiotics 1986, 39, 162. (b) Grafe, U.; Schade, W.; Fleck, W. Ger East 1986, DD 241, 74924 Dec.; Chem. Abstr. 1986, 107, 216174k.

24. Breinholt, J.; Demuth, H.; Heide, M.; Jensen, G. W.; Moller, I. L.; Nielsen, R. I.; Olsen, C. E.; Rosendahl, C. N. Acta . Chem. Scand. 1996, 50, 443.

25. Glover, V.; Bhattacharya, S. K.; Sandler, M. Indian J. Exp. Biol., 1991, 29, 1.

26. Sridhar, S. K.; Pandeya, S. N.; Stables, J. P.; Ramesh, A. Eur. J. Pharm. Sci. 2002, 16, 129.

27. Mudler, N. Swiss 1976, 580673 15.; Chem. Abstr. 1976, 86, 6388e.

28. Kubo, R. JP 328156, 1991, pp 1. 